\title{
Phosphoinositides break microtubule dynamics symmetry in the phragmoplast
}

Andrei Smertenko

Institute of Biological Chemistry, College of Human, Agricultural, and Natural Resource Sciences, Washington State University, Pullman, WA-99164, USA

e-mail: andrei.smertenko@wsu.edu

\begin{abstract}
Plant cytokinesis relies on asymmetric behaviour of microtubules - bulk polymerization at the phragmoplast leading zone and bulk depolymerization at the phragmoplast lagging zone. Recent findings demonstrate that phosphatidylinositol 4-kinase $\beta$ (PI4K $\beta$ ) plays an essential role in this asymmetry by facilitating establishment of the phragmoplast lagging zone.
\end{abstract}

Keywords: phragmoplast, microtubules, asymmetry, cell plate, phosphatidylinositol 4-kinase.

Plant cytokinesis depends on the centrifugal expansion of a specialized microtubule structure, the phragmoplast (Figure 1A,B). The expansion is driven by asymmetry of microtubule dynamics whereby bulk polymerization at the phragmoplast leading zone and bulk depolymerization at the lagging zone occurs. Microtubule behaviour in the phragmoplast correlates with cell plate assembly stages (Figure 1A,B,D). Initial delivery and fusion of cytokinetic vesicles (Stage I) is accompanied by establishment of the anti-parallel microtubule overlap. During Stage II (the tubulovesicular network), the overlaps disappear and microtubule ends terminate at the ribosomefree cell plate assembly matrix. Consolidation of membranes into the tubular network during Stage III triggers microtubule depolymerization. The subsequent two stages, formation of the fenestrated sheet (Stage IV) and cell plate maturation that results in a functional cross-wall (Stage V), occur without microtubules [1, 2].

Phragmoplast expansion necessitates coordination between the processes of microtubule dynamics (polymerization-depolymerization), membrane trafficking, and the deposition of oligosaccharides in the phragmoplast midzone [1]. However, the mechanism by which microtubule organization perceives transitions between cell plate assembly stages remains a mystery. 
Experimental data suggest the existence of a 'quality control mechanism' in the cell plate that prevents premature microtubule depolymerization [reviewed by 3]. For example, cell plate assembly failure following treatment with inhibitors of exocytosis (Brefeldin A) or vesicle fusion (caffeine) prevents depolymerization of microtubules, resulting in larger, disk-shaped phragmoplasts [reviewed by 3, 4]. In contrast, ectopic stabilization of microtubules with taxol reduces the rate of phragmoplast expansion, but does not prevent microtubule depolymerization in the distal zone [5]. Therefore, the asymmetry of microtubule dynamics and organization could be governed by the chemical composition of the cell plate.

Lipid composition in the cell plate may act as a potential regulator of microtubules in the phragmoplast. For example, mutations in enzymes that are responsible for sterol biosynthesis impair cell plate assembly - such as sterol methyltransferase smt1/cph [6] and cyclopropylsterol isomerase1-1 cpi1-1 [7]. Inhibiting the synthesis of long-chain sphingolipids with Fumonisin B1 also prevents the fusion of cytokinetic vesicles [8].

Recent work by Lin et al. [9] demonstrates the importance of phosphatidylinositol phosphates in phragmoplast asymmetry. A double knockout of phosphatidylinositol 4-kinase $\beta$ (PI4K $\beta$ ), pi4k $\beta 1$ pi4k $\beta 2$, results in cytokinetic failure and appearance of multi-nucleated cells (ca. $10 \%$ ) in the root apical meristem. Electron microscopy revealed an accumulation of vesicles in the midzone that apparently failed to fuse. These results indicate that the product of PI4K $\beta$, phosphatidylinositol 4,5-bisphosphate (Ptdlns(4,5)P2), plays a role in establishing the tubulovesicular network during Stage II of cell plate assembly.

According to the current cytokinesis model (Figure 1D), inhibiting cytokinetic vesicle fusion in the leading zone would slow cytokinesis and prevent dissolution of the anti-parallel microtubule overlaps. Consistent with this prediction, Lin et al. report slower phragmoplast expansion in pi4k $\beta 1$ pi4k $\beta 2$ and persistence of the anti-parallel microtubules marker MAP65-3 through the midzone of the expanding phragmoplast. These findings mean that (i) cell plate in pi4k $\beta 1$ pi4k $\beta 2$ fails to produce the signal for dissolution of the overlaps; and (ii) Ptdlns(4,5)P2-dependent processes in the phragmoplast act upstream of the CDK, MAPK, and Aurora kinase that collectively control binding of MAP65 to microtubules (Figure 1D). Furthermore, these findings provide further experimental support for the existence of checkpoints in cell plate assembly, which prevents premature microtubule reorganization in the midzone. 
In addition to slowed expansion, phragmoplast microtubule depolymerization was inhibited in pi4k $\beta 1$ pi $4 k \beta 2$ cells resulting in large, disk-shaped phragmoplasts that resembled those induced by treatment with Brefeldin A or caffeine [reviewed in 3,4]. This phenotype indicates a failure to establish the phragmoplast lagging zone. Hence, inhibiting the assembly of the tubulovesicular network prevents destabilization of the anti-parallel microtubule overlaps and all downstream cell plate assembly stages (Figure 1C,D). Interestingly, however, even with a failure to remodel the cell plate, delivery of the cytokinetic vesicles is not disrupted and the phragmoplast continues to expand (Figure 1C). These findings indicate that: (i) the production of the cytokinetic vesicles is independent of cell plate assembly rate; (ii) the rate of phragmoplast expansion is limited by membrane fusion and remodeling rather than by the availability of the cytokinetic vesicles; and (iii) Ptdlns $(4,5) P 2$ is not essential for the delivery of vesicles to the midzone.

The key question remains: how does Ptdlns(4,5)P2 contribute to assembly of the cell plate? Phosphoinositides could rigidify the plasma membrane and in doing so provide a counterforce for the conformational changes of dynamin that facilitate clathrin-dependent endocytosis [10]. Dynamin-related proteins decorate dumbbell-shaped membrane compartments at the phragmoplast leading edge [1]. Plausibly, Ptdlns(4,5)P2 facilitates vesicle fusion and the subsequent dynamin-dependent remodelling during assembly of the tubulovesicular network.

In conclusion, PI4K $\beta 2$ and its product Ptdlns(4,5)P2 appear to play a role in the tubulovesicular network assembly. However, careful structural analysis of the cell plate in pi4k $\beta 1$ pi4k $\beta 2$ is necessary to test this hypothesis. Future work on the pi4k $\beta 1$ pi4k $\beta 2$ mutant would facilitate understanding the role of cross talk between cell plate assembly and microtubule organization in the generation and maintenance of phragmoplast asymmetry.

\section{Acknowledgements}

This work was supported by National Science Foundation CAREER award \#1751204 and USDA-NIFA hatch project WNP00826. The author is very grateful to Dr. Mark Hubbard for editing the manuscript. I apologize to all authors whose primary research could not be cited here due to space limitations. 


\section{Figure legend}

\section{Figure 1. Phragmoplast expansion in control and pi4k $\beta 1$ pi4k $\beta 2$ mutant cells.}

A, During phragmoplast initiation anti-parallel microtubule overlaps form in all parts of the central spindle. Cytokinetic vesicles accumulate and fuse in this region, giving rise to the partition between daughter cells, the cell pate. The central spindle gives rise to the phragmoplast midzone.

B, During phragmoplast expansion cytokinetic vesicles coalesce in the phragmoplast leading zone. Some microtubules form anti-parallel overlaps. Vesicle fusion results in a tubulovesicular network surrounded by the cell plate assembly matrix. Next, cell plate assembly progresses to the tubular network stage, which triggers bulk microtubule depolymerization.

C, The tubulovesicular network fails to form in the expanding phragmoplast of pi4k $\beta 1$ pi $4 k \beta 2$ and the lagging zone is not established. The phragmoplast expands, but the anti-parallel microtubule overlaps persist throughout the midzone. Consequently, cytokinesis in the mutant culminates with the cell wall stubs instead of the cross-wall.

D, MAP65 stabilizes anti-parallel microtubule overlaps, which recruit cytokinetic vesicles with the aid of the tethering complex TRAPPII and the syntaxin KNOLLE. Vesicles fuse and undergo remodelling through the activity of dynamin-related proteins (DRP); PI4K $\beta 2$ could facilitate vesicle fusion and remodeling by a Ptdlns(4,5)P2-dependent increase of membrane rigidity. PI4Kß2 acts upstream of the signal that causes dissolution of the anti-parallel overlaps. Vesicle fusion promotes MAP65 phosphorylation by MAPK, CDK, and Aurora kinases; phosphorylated MAP65 dissociates from the microtubules; microtubules are stabilized through interaction with the cell plate assembly matrix. Establishment of the tubular network and deposition of oligosaccharides to the cell plate lumen (mostly callose) triggers depolymerization of microtubules. 


\section{References}

1. Segui-Simarro JM, Otegui MS, Austin JR, 2nd, Staehelin LA. Plant cytokinesis insights gained from electron tomography studies. In: Verma DPS, Hong Z, editors. Cell Division Control in Plants. 9. Berlin Heidelberg: Springer-Verlag; 2007. p. 251-87. 2. Smertenko A, Assaad F, Baluska F, Bezanilla M, Buschmann H, Drakakaki G, et al. Plant Cytokinesis: Terminology for Structures and Processes. Trends Cell Biol. 2017;27:885-94. doi: 10.1016/j.tcb.2017.08.008. PubMed PMID: 28943203.

3. Smertenko A, Hewitt SL, Jacques CN, Kacprzyk R, Liu Y, Marcec MJ, et al. Phragmoplast microtubule dynamics - a game of zones. J Cell Sci. 2018;131(2). doi: 10.1242/jcs.203331. PubMed PMID: WOS:000424842400009.

4. Yasuhara $\mathrm{H}$, Shibaoka $\mathrm{H}$. Inhibition of cell-plate formation by brefeldin A inhibited the depolymerization of microtubules in the central region of the phragmoplast. Plant Cell Physiol. 2000;41(3):300-10. PubMed PMID: WOS:000086051400007.

5. Yasuhara H, Sonobe S, Shibaoka H. Effects of taxol on the development of the cell plate and of the phragmoplast in tobacco BY-2 cells. Plant Cell Physiol. 1993;34(1):21-9. PubMed PMID: WOS:A1993KK58300003.

6. Schrick K, Fujioka S, Takatsuto S, Stierhof YD, Stransky H, Yoshida S, et al. A link between sterol biosynthesis, the cell wall, and cellulose in Arabidopsis. Plant J. 2004;38(2):227-43. doi: 10.1111/j.1365-313X.2004.02039.x. PubMed PMID: 15078327. 7. Men S, Boutte Y, Ikeda Y, Li X, Palme K, Stierhof YD, et al. Sterol-dependent endocytosis mediates post-cytokinetic acquisition of PIN2 auxin efflux carrier polarity. Nat Cell Biol. 2008;10(2):237-44. doi: 10.1038/ncb1686. PubMed PMID: 18223643.

8. Molino D, Van der Giessen E, Gissot L, Hematy K, Marion J, Barthelemy J, et al. Inhibition of very long acyl chain sphingolipid synthesis modifies membrane dynamics during plant cytokinesis. Biochim Biophys Acta. 2014;1842(10):1422-30. doi: 10.1016/j.bbalip.2014.06.014. PubMed PMID: 25004376.

9. Lin F, Krishnamoorthy P, Schubert V, Hause G, Heilmann M, Heilmann I. A dual role for cell plate-associated PI4Kbeta in endocytosis and phragmoplast dynamics during plant somatic cytokinesis. EMBO J. 2019. doi: 10.15252/embj.2018100303. PubMed PMID: 30617084.

10. Heilmann M, Heilmann I. Plant phosphoinositides-complex networks controlling growth and adaptation. Biochimica Et Biophysica Acta-Molecular and Cell Biology of Lipids. 2015;1851(6):759-69. doi: 10.1016/j.bbalip.2014.09.018. PubMed PMID: WOS:000353095800007. 
A

Phragmoplast initiation
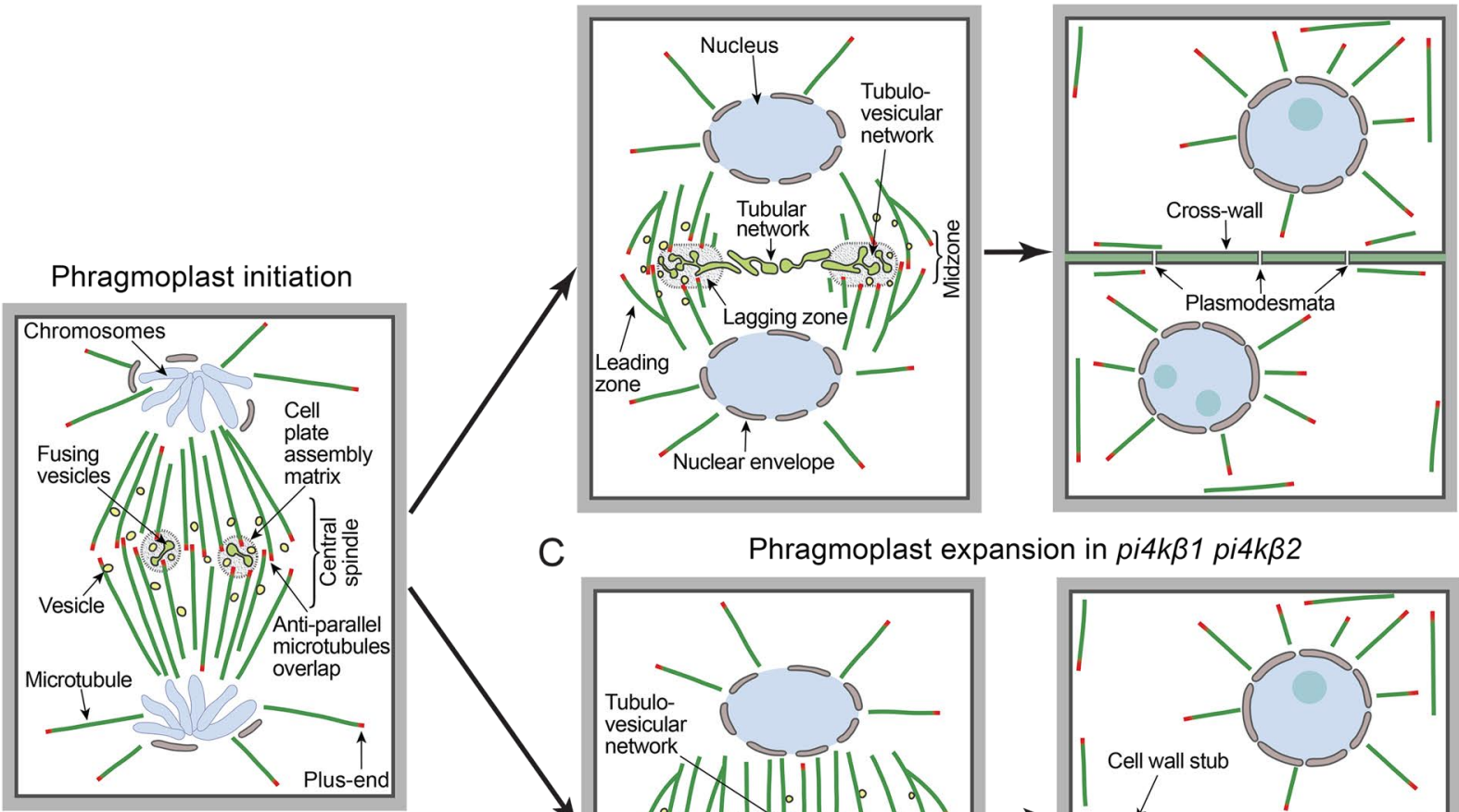

C
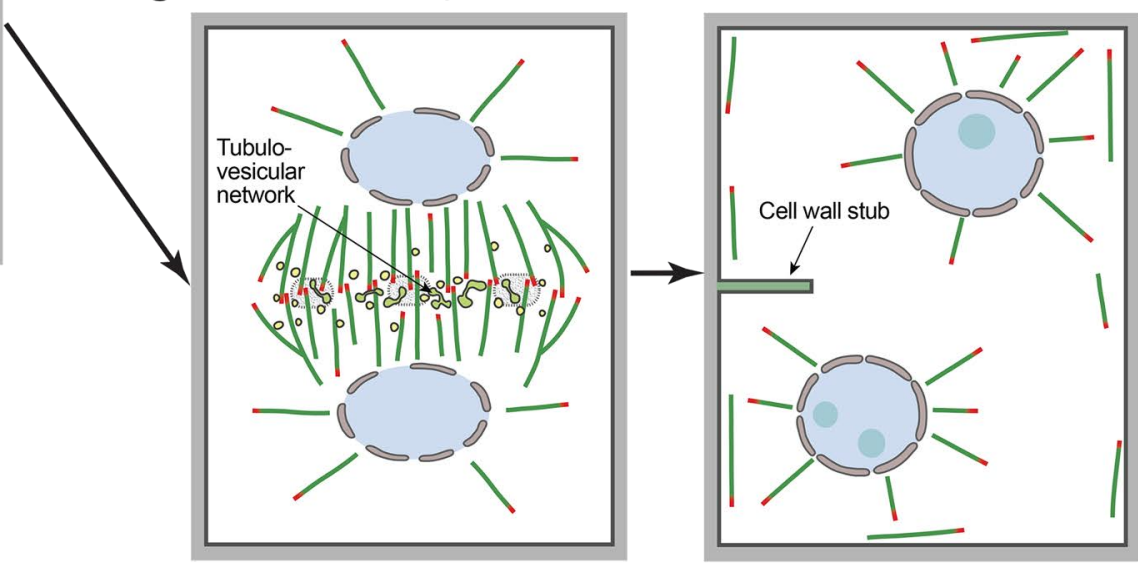

D Establishment
of microtubule

Recruitment of cytokinetic overlaps

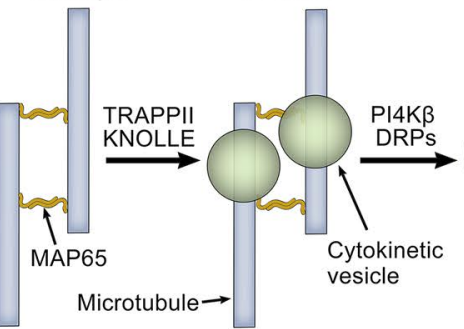

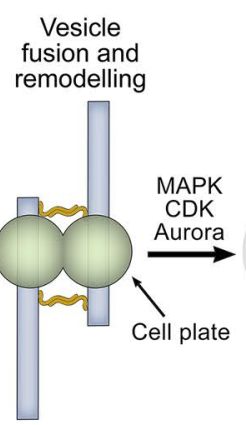

Depolymerization of microtubules

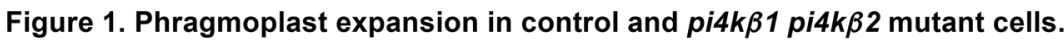

A, During phragmoplast initiation anti-parallel microtubule overlaps form in all parts of the central spindle. Cytokinetic vesicles accumulate and fuse in this region, giving rise to the partition between the daughter cells, the cell pate. The central spindle gives rise to the phragmoplast midzone.

B, During phragmoplast expansion cytokinetic vesicles coalesce in the phragmoplast leading zone. Some microtubules form anti-parallel overlaps. Vesicle fusion results in tubulovesicular network surrounded by the cell plate assembly matrix. Next, cell plate assembly progresses to the tubular network stage, which triggers bulk microtubule depolymerization.

C, The tubulovesicular network fails to form in expanding phragmoplast of pi4k $\beta 1$ pi4k $\beta 2$ and the lagging zone does not establish. The phragmoplast expands, but the anti-parallel microtubule overlaps persist throughout the midzone. Consequently, cytokinesis in the mutant culminates with the cell wall stubs instead of the cross-wall.

D, MAP65 stabilizes anti-parallel microtubule overlaps, which recruit cytokinetic vesicles with the aid of tethering complex TRAPPII and syntaxin KNOLLE. Vesicles fuse and undergo remodelling through the activity of dynamin-related proteins (DRP); PI4Kß2 could facilitate vesicle fusion and remodeling by Ptdlns (4,5)P2-dependent increase of membrane rigidity. PI4K 32 acts upstream of the signal that causes dissolution of the anti-parallel overlaps. Vesicle fusion promotes MAP65 phosphorylation by MAPK, CDK, and Aurora kinases; phosphorylated MAP65 dissociates from the microtubules; microtubules are stabilized through interaction with the cell plate assembly matrix.

Establishment of the tubular network and deposition of oligosaccharides to the cell plate lumen (mostly callose) triggers depolymerization of microtubules. 